\section{Melanie Böttger}

Graf, Susanne: Verdachts- und ereignisunabhängige Personenkontrollen - Polizeirechtliche und verfassungsrechtliche Aspekte der Schleierfahndung, Dissertation Universität Freiburg 2004, Schriften zum Öffentlichen Recht, Band 1016, 414 Seiten, Duncker \& Humblot, Berlin 2006, ISBN 3-428-11822-7

Diese Dissertation wurde im November 2004 von der rechtswissenschaftlichen Universität Freiburg angenommen und befindet sich auf dem aktualisierten Stand Mai 2005. Susanne Graf promovierte mit der vorliegenden Arbeit in einem Themengebiet, in dem Mitte der 90er Jahre eine Kontroverse auf der Forschungsebene entbrannte (S. 40). Ihre Dissertation ist derzeit das aktuellste Werk im Bereich der Schleierfahndung.

In der Einleitung geht die Verfasserin auf den rechtspolitischen Begriff der Schleierfahndung ein und erörtert seine begriffliche Unschärfe für die Rechtswissenschaft. Sie sieht in dem rechtswissenschaftlich unklaren, rechtspolitisch geprägten Begriff den Vorteil, sich von rechtlichen Präferenzen losreißen und diese überwinden zu können, um eigene, rechtswissenschaftliche Wege einschlagen und gehen zu können. Der Begriff "Schleierfahndung" ist der gebräuchliche Begriff für polizeiliche Informationsgewinnung, die in der Literatur zum Beispiel auch als „verdachts- und ereignisunabhängige Maßnahme" bezeichnet wird. Er soll deutlich machen, dass es nicht um punktuelle, anlassbezogene Maßnahmen geht, sondern gewissermaßen um einen Schleier, ein dichtes Netz von Kontrollmöglichkeiten (S. 24).

Die Dissertation gliedert sich danach in fünf Teile. Im ersten Teil (S. 32- 97) werden die normativen Grundlagen der Schleierfahndung und ihre rechtliche Einordnung angesprochen. Der zweite Teil (S. 98- 214) widmet sich den polizeirechtlichen Aspekten, während sich der dritte Teil (S. 215-340) mit den verfassungs- und europarechtlichen Aspekten der Schleierfahndung auseinandersetzt. Diese beiden Teile bilden die Schwerpunkte der Arbeit. Der vierte Teil (S. 341-367) beschäftigt sich mit den sonstigen Aspekten der Schleierfahndung; zum Beispiel werden die Vereinbarkeit mit dem Schengener Durchführungsübereinkommen und mit der Europäischen Konvention zum Schutze der Menschenrechte und Grundfreiheiten sowie die Rechtsschutz- möglichkeiten aufgezeigt. Am Ende der Arbeit wird eine Zusammenfassung (S. 368375) gegeben.

Den datenschutzrechtlichen Aspekt der Arbeit greift die Autorin auf, indem sie auf Datenverarbeitungsmaßnahmen eingeht (S. $143 \mathrm{ff}$., $182 \mathrm{ff}$.) und diese nur dann für zulässig erachtet, „wenn die Vermutung besteht, dass die personenbezogenen Daten in $\mathrm{Zu}$ kunft zur Gefahrenabwehr benötigt werden können" (S. 144). Es wird ein konkreter Anlass zur Gefahrenvorsorge gefordert. Die Datenspeicherung wird insofern unter den Vorbehalt der Erforderlichkeit gestellt (S. 145). Eine Befugnis zur verdachts- und ereignislosen Datenverarbeitung wird verneint (S. 144). Der Datenabgleich mit den Fahndungsdateien "Personenfahndung" und "Sachfahndung" (INPOL und SIS) ist hinsichtlich der in der Dissertation untersuchten Befragungs- und Identitätsfeststellungsnormen und unter der Prämisse des Zweckbindungsansatzes (S. 148, 183) - den Dissertationstitel wiederspiegelnd - verdachts- und ereignisunabhängig zulässig (S. 145-161), soweit nicht eine abweichende Regelung wie die des Gesetzes über die öffentliche Sicherheit und Ordnung des Landes Mecklenburg- Vorpommern besteht (S. 145). Äußerst aufschlussreich sind die im Zusammenhang mit einer Zweckänderungsbefugnis gemäß $\S 481$ StPO erfolgten Aussagen zu dessen Verfassungskonformität (S. 150-157). Nicht umfasst wird von der Schleierfahndung ein Datenabgleich mit sonstigen Dateien wie KAN, PIOS oder PAD aus INPOL, da dieser nur verdachtsabhängig erfolgen kann und im Wege einer Schleierfahndung, die im Gegensatz dazu kraft Natur der Sache verdachtsunabhängig durchgeführt wird, unzulässig ist (S. 157 f.). Außerordentlich differenzierend wird der Frage nach der Erlaubnis von Halterabfragen anhand des Autokennzeichens des angehaltenen Fahrzeugs und von automatisierten Kennzeichenerkennungssystemen behandelt und im Ergebnis wird die Zulässigkeit verneint, da diese primär einer Sachfahndung dienen, während bei der Schleierfahndung primär die Personenkontrolle im Vordergrund steht (S. 161).

Eine Auskunftspflicht wird an das Befragungsrecht der Polizei nicht geknüpft, sondern vielmehr rigoros verneint und nur in den normierten Fällen des Gesetzes über den Bundesgrenzschutz (S. 51) und des Gesetzes über die öffentliche Sicherheit und
Ordnung des Landes Sachsen- Anhalt (S. 55) angenommen (S. $134 \mathrm{ff}$.).

Weiterhin steht das Grundrecht auf informationelle Selbstbestimmung gemäß Art. 2 Abs. 1 GG i. V. m. Art. 1 Abs. 1 GG im Raum der verfassungsrechtlichen Betrachtung bei der Frage der Verhältnismäßigkeit im weiteren Sinne (S. 276- 323). Diese Arbeit wertet den Stellenwert des Grundrechts auf informationelle Selbstbestimmung auf, indem dieses als ein wichtiges Schutzgut anerkannt wird. Die Verfasserin schränkt die Schleierfahndung zugunsten des bedeutenden Bürgerrechts ein, indem sie eine Verhältnismäßigkeit im engeren Sinne nur bejaht, wenn eine Kontrolle zu den in den Normen genannten Zwecken stattfindet. Dies sei ausschließlich dann der Fall, wenn Lageerkenntnisse auf das Vorliegen grenzüberschreitender Kriminalität hindeuten (S. $116 \mathrm{ff}$.). Die Verfasserin konstatiert, dass das so genannte "Lagebilderfordernis" nicht explizit in der jeweiligen Norm geregelt sein muss, sondern es der jeweiligen Schleierfahndungsnorm in verfassungskonformer Anwendung des Verhältnismäßigkeitsprinzips immanent sei (S. 373 f.). Unterstützung von meiner Seite wird im Hinblick auf die Forderung der Verfasserin zuteil, dass „eine Pflicht zu einer aussagekräftigen Evaluierung der ergriffenen Schleierfahndungsmaßnahmen normiert werden" müsste (S. $314 \mathrm{ff}$.).

Das Ziel der Arbeit ist die Beantwortung der Frage, ob die Schleierfahndung den besonderen rechtlichen Anforderungen genügt, die an Maßnahmen der Informationsgewinnung zu stellen sind (S. 31). Dieses Ziel wird durch eine umfangreiche und detaillierte Darstellung der besonderen rechtlichen Anforderungen an Maßnahmen zur Informationsgewinnung erreicht.

Angesichts der von Schäuble aktuell aufgeworfenen kriminalpolizeilichen Machtfrage und der damit einhergehenden Frage der Bekämpfung von grenzüberschreitender Kriminalität (S. 123 ff.) anlässlich der Migrationsbewegung (S. 26, 126 ff.) verliert die vorliegende Arbeit mitnichten an Aktualität. Dieses Werk bedeutet einen Meilenstein für das rechtswissenschaftliche Feld der Schleierfahndung und bricht eine Lanze für das informationelle Selbstbestimmungsrecht. Das Buch stellt eine Bereicherung für jede deutschsprachige, juristische Bibliothek dar. 